\title{
Detection \& Classification of Cardiac Arrhythmia
}

\author{
Chetan M. Jadhav ${ }^{1}$, Dr. V. K. Bairagi ${ }^{2}$ \\ ${ }^{1}$ Electronics Engineering Department, AISSMS's Institute of Information Technology, Pune-411001, India \\ ${ }^{2}$ E \& TC Engineering Dept, AISSMS's Institute of Information Technology, Pune-411001, India
}

\begin{tabular}{l} 
Article Info \\
\hline Article history: \\
Received Jan 18, 2017 \\
Revised Feb 28, 2017 \\
Accepted Mar 20, 2017 \\
\hline
\end{tabular}

\section{Keywords:}

Arrhythmia

Electrocardiogram

\begin{abstract}
The term Arrhythmia refers to any change from the normal sequence in the electrical impulses. It is also treated as abnormal heart rhythms or irregular heartbeats. The rate of growth of Cardiac Arrhythmia disease is very high \& its effects can be observed in any age group in society. Arrhythmia detection can be done in many ways but effective \& simple method for detection \& diagnosis of Cardiac Arrhythmia is by doing analysis of Electrocardiogram signals from ECG sensors. ECG signal can give us the detail information of heart activities, so we can use ECG signals to detect the rhythm \& behaviour of heart beats resulting into detection \& diagnosis of Cardiac Arrhythmia. In this paper new \& improved methodology for early Detection \& Classification of Cardiac Arrhythmia has been proposed. In this paper ECG signals are captured using ECG sensors \& this ECG signals are used \& processed to get the required data regarding heart beats of the human being $\&$ then proposed methodology applies for Detection \& Classification of Cardiac Arrhythmia. Detection of Cardiac Arrhythmia using ECG signals allows us for easy \& reliable way with low cost solution to diagnose Arrhythmia in its prior early stage.
\end{abstract}

Copyright $\odot 2017$ Institute of Advanced Engineering and Science. All rights reserved.

\section{Corresponding Author:}

V. K. Bairagi,

Associate Professor, E \& TC Engineering Department,

AISSMS's Institute of Information Technology,

Pune-411001, India

Email: vbairagi@yahoo.co.in

\section{INTRODUCTION}

Nowadays cardiac related diseases are increasing so rapidly in all of the age group across the world. On the basis of previous studies it has already proven $\&$ concluded that most of the deaths are not happening because of heart attacks but it is happening because of the improper heart beat rhythms that is because of Cardiac Arrhythmia hence causing this as a saviour problem commonly observed now a days. Arrhythmia refers to any type of change in heart rhythm pattern from the normal rhythm of electrical impulses [1]. In the case of Arrhythmia electrical pulse can be very fast, very slow or irregular in nature than the normal pulses. When our heart does not beat properly then it is unable to pump out blood effectively. This abnormal behaviour causes many uncertain deaths. As cardiac arrhythmia does not show any specific symptoms hence it is very difficult to track Cardiac Arrhythmia in its early stage. Hence our proposed methodology helps in early detection \& classification of Cardiac Arrhythmia which will ultimately can became a superior solution in detection of Arrhythmia in its early stage.

The person having Arrhythmia may show following types of heart beat rhythms:

a. Too slowly (Bradycardia)

b. Too quickly (Tachycardia)

c. Irregular or skipping beat rhythms

Basically Arrhythmia is a problem regarding heartbeat rhythms or heart beat rate. This means that you are heart is beating too quick or too slow. If heart beats faster than 100 beats per minute then it is known as Tachycardia. If heart beats per minute are slower than 60 beats per minute then it is known as Bradycardia. 
Normal heart beat rate is between 60 beats per minute to 100 beats per minute. Atrial Fibrillation(AF) is the very commonly found Arrhythmia, which results into quick \& uneven heart beats. This is preliminary classification of Cardiac Arrhythmia disease [1][2]. In day to day life, many other factors like heart attack, smoking and stress can cause change in heart rhythm. Sometimes arrhythmia may cause because of specific medicines consume previously. Arrhythmia can be cured by taking treatments in its early stages which may include medicines and treatments, pacemaker or medical surgery [9].
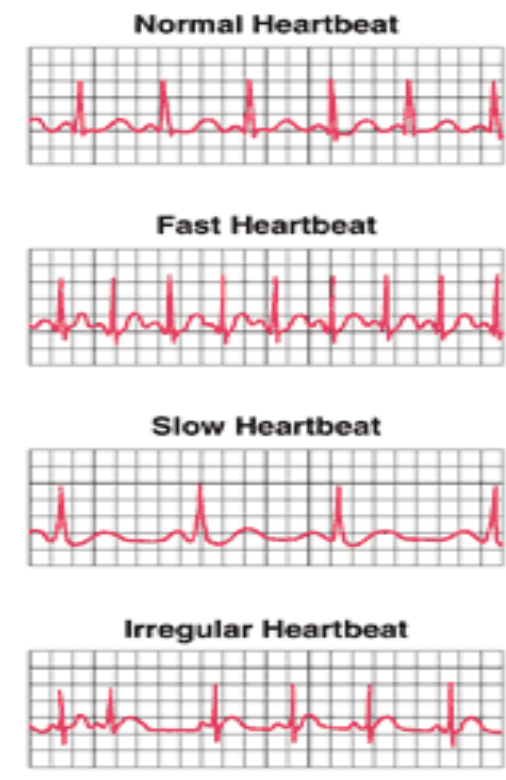

Figure 1: Various Heart beats [10]

As shown in Figure 1, various heart beat sequences, this are various types of signals captured from ECG sensors. As we know the heart of healthy \& fit person beats with a heart beat rate of 60 to 100 beats per minute, while quicker heart beat beats with heart beat rate greater than around 100 heart beats per minute with minimal R-R interval. Slow heart beat beats with heart beat rate less than 60 heart beats per minute with maximum R-R interval [9]. With the help of this heart beats per minutes \& its beating sequences, we are going to detect \& diagnose the Cardiac Arrhythmia \& its further classification.

\section{LITERATURE SURVEY}

H. Azucena et al. [1] has proposed a method for Detection of Cardiac Arrhythmia. They are using ECG signals for the detection of Cardiac Arrhythmia. Analysis of ECG signals has been done to extract some parameters from this incoming ECG signals from ECG sensors. The authors have done detailed analysis of captured ECG signals \& the extracted parameters from this ECG signals. ECG signal provides various types of parameters like heart beats per minute, heart beat sequences, R-R period etc. they have proposed their designed system on this such that they are capturing the ECG signals from human body \& parameters are getting extracted from his signals. Then this signal gets compared with the database signals which are locally stored on the computer machine in database format.

Jo Woon Chong et al. [4] has proved that algorithm can be run on smart phone which will help to detect the presence of Cardiac Arrhythmia in a patient. As now a day's smart phones are widely used in our society so this technique can become very easy, cheap \& convenient to use for the user. In this proposed technique, authors has used camera from smart phone to record the pulsatile signals from the finger tip of the patient \& then processing on this video further will gives us the results regarding the presence of Cardiac Arrhythmia in that individual or not. Authors has used technique such that they are capturing the fluctuating pulsatile signals from finger tip \& they are correlating this captured signals with heat beat signals to detect the presence of Cardiac Arrhythmia. 
With the help of the detailed literature survey we came to know about the current proposed system designs on detection of Cardiac Arrhythmia. From this survey we have modified \& proposed our new designed cost effective, reliable \& easy to use system which will be available in society with low cost.

\section{RESEARCH METHOD}

In this proposed application with the help of ECG sensors which are going to be placed on human body we are going to capture ECG signals, analysis of ECG signals will give us the heart beats rate \& with the help of this detected heart beat rate our proposed system will detect the presence of Cardiac Arrhythmia $\&$ its further classification depending upon the signals captured from the human body [1].

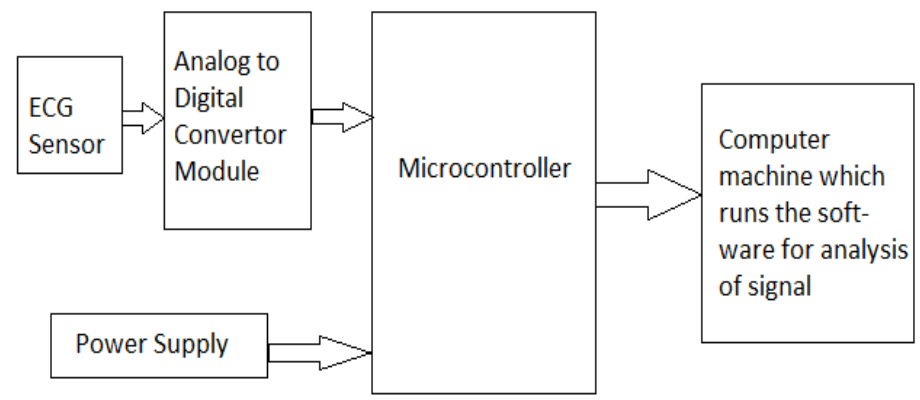

Figure 2: Block diagram of proposed System

Following are the major blocks from the system:
a. ECG sensors
b. Analog to digital converter
c. Micro controller
d. Computer machine

As shown in Figure 2 we are going to apply ECG sensors on human body which will capture the ECG signals. This ECG signals will go to analog to digital converter from the micro controller section. We are going to use 12 bit on chip ADC. ADC will give us the digitally converted output signals of ECG sensors and this digitally converted data we will pass to laptop machine over serial to USB port. This digitally converted data will get receive in MATLAB software over USB port. This continuously coming data over USB port is going to get plotted in MATLAB software. This digitally converted data coming from ECG sensors is going as a input to our proposed methodology designed in MATLAB software. With the help of detected heart beats per minute we are going to take decision whether Arrhythmia is present or not and if present then we are going for further analysis \& classification of this detected Arrhythmia.

Table 1: R-R Interval of Heart beats per minute

\begin{tabular}{ccc}
\hline Heart Arrhythmia & $\begin{array}{c}\text { Heart Beat } \\
\text { Rate(Beats/min) }\end{array}$ & R-R Interval \\
\hline Normal Case & $60-100$ & $0.60-1$ \\
Bradycardia & $<60$ & $>1$ \\
Tachycardia & $>100$ & $<0.6$ \\
\hline
\end{tabular}

As shown in Table 1, the R-R interval to be monitored for normal case is between 0.60 second to 1 second. The R-R interval for Bradycardia should be greater than 1 second to satisfy the heart beat rate condition of heart beats are greater than 60 BPM. Similar way for Tachycardia R-R interval should be less than 0.6 seconds to satisfy the heart beat condition which is BPM should be greater than 100 BPM as shown in Table 1. 


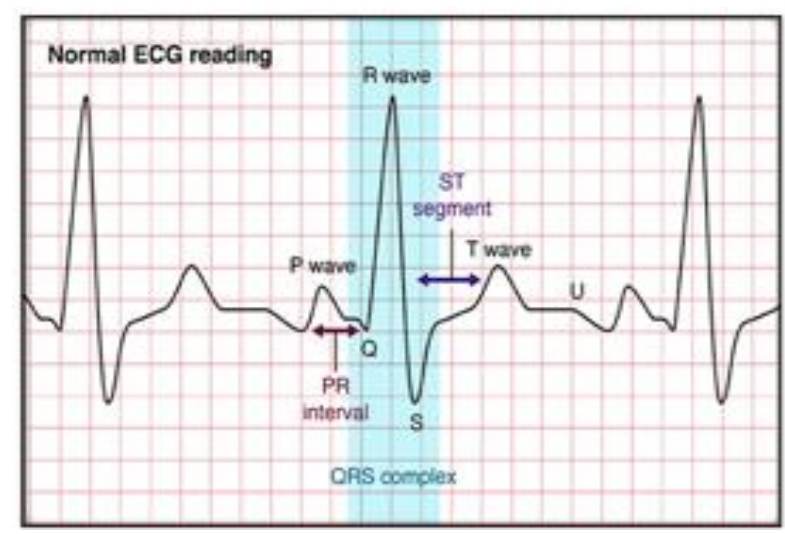

Figure 3: ECG wave details [8]

Detailed ECG PQRS points are highlighted in Figure 3, PQRS wave is having only one highest peak point known as $\mathrm{R}$ point or $\mathrm{R}$ wave. This $\mathrm{R}$ point gives information regarding the heart beats \& we are going to calculate heart beats using the calculation of time interval between consecutive $\mathrm{R}$ to $\mathrm{R}$ wave intervals [8].

\section{RESULTS AND ANALYSIS}

During this work we have developed automated electronic device to detect whether Cardiac Arrhythmia is present or not $\&$ if it is present then it will classify that detected Arrhythmia depending upon its subcategories. Our proposed system takes inputs either from ECG sensors or locally stored data base.

As shown in Figure 4, signals coming from ECG sensors are plotted over output display window in MATLAB software with some of its filtering stages. With the help of these captured signals our algorithm calculates the R-R wave interval between two consecutive waves.

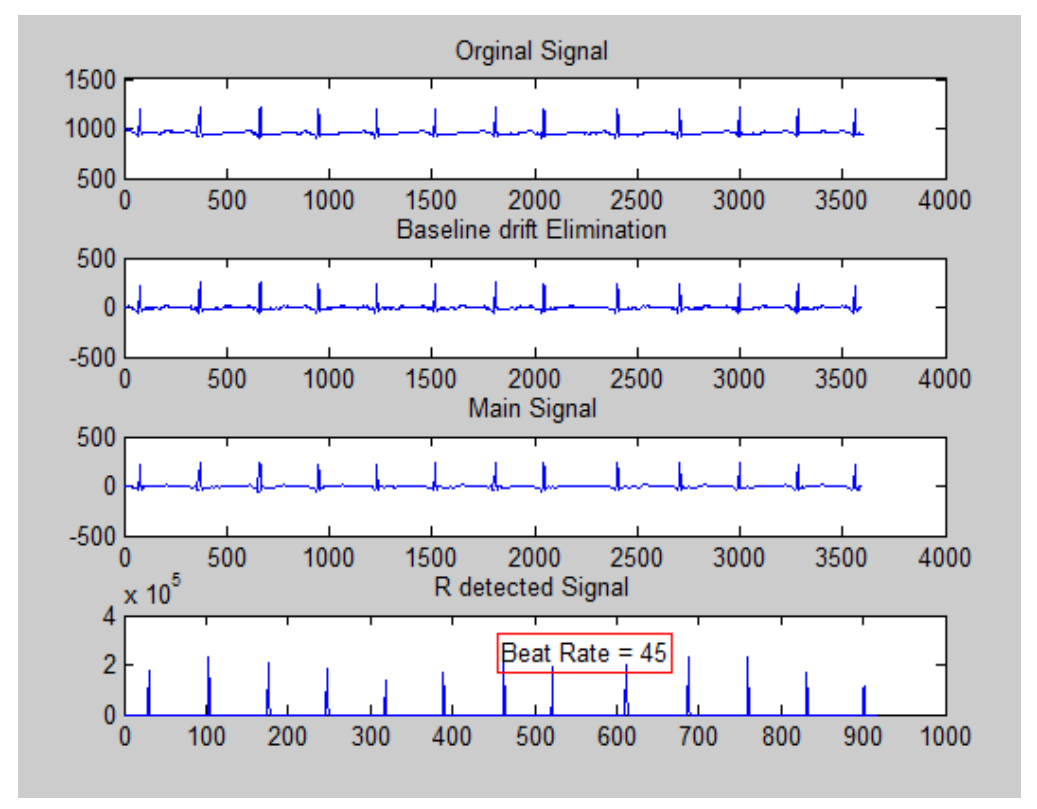

Figure 4: Captured ECG signal [11]

Depending upon the captured signals our system will calculate the heart beats per minute \& it will give us the dialogue box with total heart beats per minute as shown in Figure 5. 


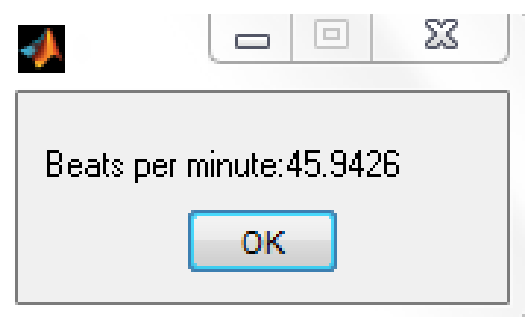

Figure 5: Beats per Minute [11]

As shown in Figure 5, total heart beats per minute count is displayed after analyzing the incoming heart beat signals from ECG sensors.

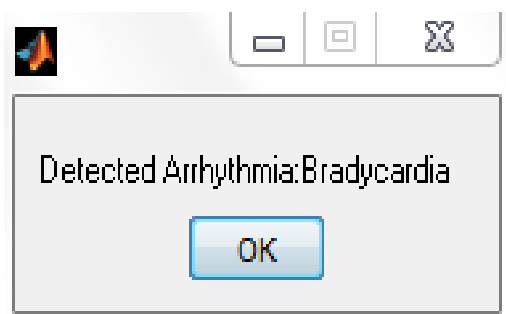

Figure 6: Classification of Arrhythmia [11]

Once system will get heart beat per minute count then system will classify this detected Arrhythmia depending upon the heart beat rate count. Depending upon the heart beat rate system will classify it whether the patient is Normal or if patient is having arrhythmia then it will classify it as Bradycardia or Tachycardia depending upon the state of the heart beat signals as shown in Figure 6.

\section{CONCLUSION}

As Cardiac Arrhythmia does not show any prior or preliminary symptoms at its early stage hence it is very difficult to treat Cardiac Arrhythmia in its early stage. Hence to avoid this automated detection \& classification has become very important to diagnose Cardiac Arrhythmia in its preliminary stage. Cardiac Arrhythmia is also treated as silent killer because it is not showing any major symptoms in early stage hence it is very important to detect the Cardiac Arrhythmia in its early stage \& treat it in early stage only to avoid the further complications \& hazardous effects on human body. So our proposed system will help to detect Cardiac Arrhythmia in its early stage \& to classify it in its subcategories into Bradycardia or Tachycardia. This system will help to diagnose the Cardiac Arrhythmia in its early stage. After analyzing \& studying many of the already defined algorithms \& methods for Detection \& Classification of Cardiac Arrhythmia we have redefined \& proposed our own cost effective, efficient $\&$ easy to use system.

\section{ACKNOWLEDGEMENTS}

Authors gratefully acknowledges the Principal of the institute Dr. P. B. Mane, Prof. D. K. Shedge, Head of Electronics Engineering, Department \& entire staff of Post Graduate section of the Institute who has been involved in this whole process of research work.

\section{REFERENCES}

[1] H. Azucena a, et al. , "Design and implementation of a simple portable biomedical electronic device to diagnose cardiac arrhythmias," ScienceDirect, Sensing and Bio-Sensing Research 4, pp.1-10, 2015.

[2] J. M. Lillo-Castellano, et al. , "Symmetrical Compression Distance for Arrhythmia Discrimination in Cloud-Based Big-Data Services," Ieee Journal of Biomedical And Health Informatics, vol.19, no. 4, July 2015.

[3] L.V.Rajani Kumari, et al. , "FPGA Based Arrhythmia Detection," 3rd International Conference on Recent Trends in Computing, Procedia Computer Science 57 , pp.970 - 979, 2015.

[4] Jo Woon Chong, et al. , "Arrhythmia Discrimination Using a Smart Phone," IEEE Journal of Biomedical and Health Informatics, vol.19, no.3, May 2015. 
[5] Roland Weber, et al. , "Diagnosis and management of common fetal arrhythmias," Journal of the Saudi Heart Association 23, pp.61-66, 2011.

[6] Gabriel Nallathambi , "Integrate and Fire Pulse Train Automaton for QRS detection", IEEE Transactions on Biomedical Engineering, vol.61, no.2, February 2014.

[7] Indu Saini, et al. , "QRS detection using K-Nearest Neighbor algorithm (KNN) and evaluation on standard ECG databases," Journal of Advanced Research 4, pp.331-344, 2013.

[8] Joseph J. Oresko, et al. , "A Wearable Smartphone-Based Platform for Real-Time Cardiovascular Disease Detection Via Electrocardiogram Processing," Ieee Transactions On Information Technology In Biomedicine, vol.14, no.3, May 2010.

[9] www.nlm.nih.gov/medlineplus/arrhythmia.html

[10] http://sevencountriesstudy.com/ecg-predictors-and-coronary-heart-disease

[11] Results of heart beats per minute captured in MATLAB software for respective designed code 